\title{
Cofinal completeness of the Hausdorff metric topology
}

by

\author{
Gerald Beer (Los Angeles, CA) and Giuseppe Di Maio (Caserta)
}

\begin{abstract}
A net in a Hausdorff uniform space is called cofinally Cauchy if for each entourage, there exists a cofinal (rather than residual) set of indices whose corresponding terms are pairwise within the entourage. In a metric space equipped with the associated metric uniformity, if each cofinally Cauchy sequence has a cluster point, then so does each cofinally Cauchy net, and the space is called cofinally complete. Here we give necessary and sufficient conditions for the nonempty closed subsets of the metric space equipped with Hausdorff distance to be cofinally complete.
\end{abstract}

1. Introduction. A net $\left\langle x_{\lambda}\right\rangle_{\lambda \in \Lambda}$ in a Hausdorff uniform space $\langle X, \mathfrak{U}\rangle$ is of course called Cauchy if for each entourage $U$, there exists a residual subset $\Lambda_{0}$ of $\Lambda$ such that whenever $\{\lambda, \mu\} \subseteq \Lambda_{0}$, then $\left(x_{\lambda}, x_{\mu}\right) \in U$. Replacing residual by cofinal in the definition, we obtain nets that are called cofinally Cauchy, and those uniform spaces in which each cofinally Cauchy net clusters are naturally called cofinally complete [6, 7, 12, 14, 15, 21]. Cofinal completeness can as expected be described in terms of filters [14, 15], and from this perspective it was shown to be intrinsic to paracompactness by Corson [9]. Corson implicitly showed that a (completely regular) Hausdorff space is paracompact if and only if it admits a uniformity for which the associated uniform space is cofinally complete.

Twenty years after Corson's seminal paper appeared, Rice [20] introduced the notion of uniform paracompactness for a Hausdorff uniform space: for each open cover $\left\{V_{i}: i \in I\right\}$ of a uniform space $\langle X, \mathcal{U}\rangle$, there exists an open refinement and an entourage $U$ such that for each $x \in X, U(x)$ meets only finitely many members of the refinement. He then produced two separate descriptions of uniform paracompactness. Later it was observed that one of these descriptions was equivalent to cofinal completeness of nets: for each open cover of $X$ directed by inclusion, there exists an entourage $U$

2010 Mathematics Subject Classification: Primary 54B20; Secondary 54E35, 54E45, 54E50. Key words and phrases: Hausdorff distance, cofinal completeness, uniform paracompactness, local compactness, measure of local compactness. 
such that $\{U(x): x \in X\}$ refines the initial cover [15, 22. Subsequently, Fried and Frolík [11] characterized uniform paracompactness in terms of a functional separation property within the semiuniform product of $X$ and an arbitrary compactification of $X$.

As expected, with their additional structure, more can be said about cofinal completeness/uniform paracompactness in the context of metric spaces. For example, Hohti [13] showed that a metric space $\langle X, d\rangle$ is uniformly paracompact if and only if $(1) \operatorname{nlc}(X):=\{x \in X: x$ has no compact neighborhood $\}$ is compact, and (2) for all $\delta>0,\{x \in X: d(x, \operatorname{nlc}(X))>\delta\}$ is uniformly locally compact. Recently, Beer [6] showed that sequential cofinal completeness is equivalent to Hohti's conditions, so that for metric spaces, if each cofinally Cauchy sequence has a cluster point, then so does each cofinally Cauchy net. Outside the metric setting, that each cofinally Cauchy sequence has a cluster point does not guarantee that each net based on a well-ordered (directed) set does. Consider the ordinal space $[0, \Omega)$ where $\Omega$ is the first uncountable ordinal. Each sequence $\left\langle x_{n}\right\rangle$ in the space without restriction has a cluster point (one such is $\inf _{n}\left(\sup _{k \geq n} x_{k}\right)$ ). Now consider the trace of the unique totally bounded uniformity for the compact Hausdorff space $[0, \Omega]$ on $[0, \Omega)$. Since the induced uniformity is also totally bounded, each net $\left\langle x_{\lambda}\right\rangle_{\lambda \in \Lambda}$ in $[0, \Omega)$ is cofinally Cauchy. But the identity net fails to have a cluster point. Separately, Burdick [7, Example 3.1] has shown that so-called linear cofinal completeness for nets in a Hausdorff uniform space, where the directed sets must be well-ordered, is a properly weaker notion than cofinal completeness for arbirary nets (linear cofinal completeness evidently fails for $[0, \Omega)$ ).

Results of [6] show that the cofinally complete metric spaces seem to constitute a parallel universe to the UC-spaces [4, 5, 6, 24, also known as the Atsuji spaces [2, 3, 16] or Lebesgue spaces [18], which also forms an intermediate class of metric spaces between the compact spaces and the complete ones. Most familiarly, a metric space $\langle X, d\rangle$ is a UC-space if and only if each continuous function $f$ with values in an arbitrary metric space is uniformly continuous [1, 5]. Cofinally complete spaces can also be characterized in terms of uniform properties of continuous functions: a metric space is cofinally complete if and only if each continuous function with values in an arbitrary metric space is uniformly locally bounded [6]. The UC-spaces, too, have been shown by Toader [5, 23] to be those for which generalized Cauchy sequences (with distinct terms) in a somewhat different sense have cluster points. Both classes can be described in terms of Cantor-type theorems for decreasing sequences of closed sets [3, 6]. Both can be characterized by the existence of cluster points for sequences on which certain geometric functionals go to zero; in the case of UC-spaces, the functional measures the isolation of points of $X$ [1], whereas for cofinally complete spaces, the 
functional measures the local compactness of the space at each point [6]. A metrizable space $X$ has a compatible UC-metric if and only if its set of limit points $X^{\prime}$ is compact [4, 19, whereas it admits a compatible cofinally complete metric if and only if $\operatorname{nlc}(X)$ is compact [6, 21].

A basic question to ask in each case is this: when are the nonempty closed subsets $\mathcal{C}_{0}(X)$ of a metric space $\langle X, d\rangle$ equipped with Hausdorff distance again a space of each type? In the case of UC-spaces, the answer is utterly transparent [2, Theorem 3]: either $X$ is compact or $X$ is uniformly discrete. The purpose of this paper is to answer the much more difficult question for cofinally complete metric spaces, using results on local compactness of hyperspaces of Costantini, Levi and Pelant [10].

2. Preliminaries. All metric spaces will be assumed to contain at least two points. If $A$ is a subset of a metric space $\langle X, d\rangle$, we denote its closure and set of limit points by $\operatorname{cl}(A)$ and $A^{\prime}$, respectively. We denote the nonempty subsets of $\langle X, d\rangle$ by $\mathcal{P}_{0}(X)$ and its closed subsets including $\emptyset$ by $\mathcal{C}(X)$. If $x \in X$ and $A \in \mathcal{P}_{0}(X)$ we write $d(x, A)$ for $\inf _{a \in A} d(x, a)$. Given $\varepsilon>0$, we call $A \in \mathcal{P}_{0}(X)$ an $\varepsilon$-discrete subset if whenever $a_{1} \neq a_{2}$ in $A$, then $d\left(a_{1}, a_{2}\right) \geq \varepsilon$. If $A, B$ are nonempty subsets of $X$, we call $D_{d}(A, B):=$ $\inf \{d(a, b): a \in A, b \in B\}$ the gap between them. When $A \neq \emptyset$, we adopt the convention $D_{d}(A, \emptyset)=D_{d}(\emptyset, A)=\infty$.

If $x$ is a point of a metric space $\langle X, d\rangle$ and $\alpha>0$, we denote the open ball with center $x$ and radius $\alpha$ by $S_{d}(x, \alpha)$. We call $x$ a point of local compactness of $X$ if for some $\alpha>0, \operatorname{cl}\left(S_{d}(x, \alpha)\right)$ is compact. Following [6], if $x \in X$ has a compact neighborhood, set

$$
\nu(x)=\sup \left\{\varepsilon>0: \operatorname{cl}\left(S_{d}(x, \varepsilon)\right) \text { is compact }\right\} ;
$$

otherwise, set $\nu(x)=0$. We call a nonempty subset $A$ of $X$ uniformly locally compact if $\inf \{\nu(a): a \in A\}>0$; note that this does not imply that $A$ is even locally compact as a metric subspace of $X$. A major result of [6] says that $\langle X, d\rangle$ is cofinally complete if and only if each sequence $\left\langle x_{n}\right\rangle$ in $X$ with $\lim _{n \rightarrow \infty} \nu\left(x_{n}\right)=0$ clusters. As a uniformly locally compact space $X$ has no such sequence, the criterion is fulfilled vacuously.

If $A$ is a possibly empty subset of $X$ and $\varepsilon>0$, the $\varepsilon$-enlargement of $A$ is $A^{\varepsilon}:=\bigcup_{a \in A} S_{d}(a, \varepsilon)$. If $A, B$ are nonempty closed subsets of $X$, we define the Hausdorff distance [5, 17] between them by

$$
\begin{aligned}
H_{d}(A, B) & =\max \{\{\sup \{d(a, B): a \in A\}, \sup \{d(b, A): b \in B\}\} \\
& =\inf \left\{\varepsilon>0: B \subseteq A^{\varepsilon} \text { and } A \subseteq B^{\varepsilon}\right\} .
\end{aligned}
$$

Hausdorff distance so defined is an extended real-valued metric on $\mathcal{C}_{0}(X)$ which is finite-valued when restricted to the nonempty closed and bounded sets. Of course, $x \mapsto\{x\}$ is an isometry of $\langle X, d\rangle$ into $\left\langle\mathcal{C}_{0}(X), H_{d}\right\rangle$. While there are many ways to topologize the nonempty closed subsets of a metric 
space, we will call $\left\langle\mathfrak{C}_{0}(X), H_{d}\right\rangle$ the hyperspace of $X$ here. It is known that two equivalent metrics determine the same hyperspace if and only if they are uniformly equivalent metrics [5, Theorem 3.3.2]. Properties of completeness, total boundedness, compactness of $\langle X, d\rangle$ carry over to the hyperspace [5, Thm. 3.2.4]. Burdick [8] gave an internal condition on $\langle X, d\rangle$ characterizing local compactness of $\left\langle\mathfrak{C}_{0}(X), H_{d}\right\rangle$, and observed that local compactness of the hyperspace guarantees uniform local compactness as well, and is guaranteed by $X$ being a point of local compactness of the hyperspace. Actually, his results are stated in the more general setting of uniform spaces. Here, we rely on a more technical condition given by Costantini, Levi and Pelant [10] to describe when a particular nonempty closed set is a point of local compactness in the hyperspace.

3. When is a hyperspace cofinally complete? The main purpose of this section is to present for a metric space $\langle X, d\rangle$ necessary and sufficient conditions for the associated hyperspace $\left\langle\mathfrak{C}_{0}(X), H_{d}\right\rangle$ to be cofinally complete. In the process we give a tractable description of $\nu(C)$ for $C \in \mathcal{C}_{0}(X)$ for an arbitrary metric hyperspace. Of course formally,

$\nu(C)=\sup \left\{\varepsilon>0: S_{H_{d}}(C, \varepsilon)\right.$ has compact closure in the hyperspace $\}$.

Singleton subsets of $X$ belong to the hyperspace and fortunately for the sake of clarity we have the following result.

Proposition 3.1. Let $\langle X, d\rangle$ be a metric space and let $x_{0} \in X$ be arbitrary. Then $\nu\left(x_{0}\right)=\nu\left(\left\{x_{0}\right\}\right)$, the latter computed relative to $\mathcal{C}_{0}(X)$.

Proof. If $A \in \mathfrak{C}_{0}(X)$ and $H_{d}\left(A,\left\{x_{0}\right\}\right)<\alpha$, then $A \subseteq S_{d}\left(x_{0}, \alpha\right)$. Hence if $S_{H_{d}}\left(\left\{x_{0}\right\}, \alpha\right)$ has compact closure, then so does $S_{d}\left(x_{0}, \alpha\right)$ because $x \mapsto\{x\}$ is an isometric embedding and singleton subsets are closed in the hyperspace. This proves $\nu\left(\left\{x_{0}\right\}\right) \leq \nu\left(x_{0}\right)$. On the other hand, if $\operatorname{cl}\left(\left\{x: d\left(x, x_{0}\right\}<\alpha\right\}\right)$ is compact in $X$, then for each $\beta<\alpha,\left\{x: d\left(x, x_{0}\right\} \leq \beta\right\}$ is compact and so is the set of all of its closed subsets [5, Theorem 3.2.4], which is nothing more than $\left\{A \in \mathcal{C}_{0}(X): H_{d}\left(A,\left\{x_{0}\right\}\right) \leq \beta\right\}$. But this contains the $H_{d}$-closure of $\left\{A \in \mathcal{C}_{0}(X): H_{d}\left(A,\left\{x_{0}\right\}\right)<\beta\right\}$, and we conclude that $\nu\left(\left\{x_{0}\right\}\right) \geq \nu\left(x_{0}\right)$.

Our description of $\nu(C)$ for a nonempty closed set $C$ has as a point of departure the negation of the criterion $\left(\star_{C}^{+}\right)$that Costantini, Levi and Pelant gave [10, p. 587] for a set $C \in \mathcal{C}_{0}(X)$ to satisfy $\nu(C)>0$, which is perhaps easier to understand than the criterion itself. Structurally, their criterion $\left(\star_{C}^{+}\right)$is of the form

$$
\exists \widehat{\varepsilon}(P(\widehat{\varepsilon}) \text { and } Q(\widehat{\varepsilon})) \text {, }
$$

whose negation structurally can be presented as

$$
\forall \widehat{\varepsilon}(P(\widehat{\varepsilon}) \Rightarrow \neg Q(\widehat{\varepsilon})) .
$$


With this in mind we can present the negation of the condition $\left(\star_{C}^{+}\right)$of Costantini, Levi and Pelant in the following way:

$\neg\left(\star_{C}^{+}\right)$Whenever $\widehat{\varepsilon}$ is a positive number such that inf $\{\nu(x): x \in C\} \geq \widehat{\varepsilon}$, there exist $\varepsilon$ and $\delta$ with $0<\delta<\varepsilon<\widehat{\varepsilon}$ and an infinite $\varepsilon$-discrete subset $D$ of $C$ such that for all $x \in D, S_{d}(x, \varepsilon) \backslash S_{d}(x, \delta) \neq \emptyset$.

This negation leads us to make the following definition.

Definition 3.2. By an $\varepsilon$-satellite system for a set $C$ in a metric space $\langle X, d\rangle$ we mean the union of an infinite $\varepsilon$-discrete subset $D$ of $C$ and $\left\{y_{x}: x \in\right.$ $D\} \subseteq X$ such that for some $\delta \in(0, \varepsilon)$ and all $x \in D$ we have $\delta<d\left(x, y_{x}\right)<\varepsilon$.

In the definition, $D$ and $\left\{y_{x}: x \in D\right\}$ are automatically disjoint so that if $D_{0}$ is an infinite of subset of $D$ then $D_{0} \cup\left\{y_{x}: x \in D_{0}\right\}$ is again an $\varepsilon$-satellite system. Note also that an $\varepsilon$-satellite system in a set $C$ is again one in any superset of $C$. We also observe that the set of satellites $\left\{y_{x}: x \in D\right\}$ in the definition need not be infinite: if $C \subseteq \ell_{2}$ consists of the standard orthonormal base $\left\{e_{n}: n \in \mathbb{N}\right\}$, then $\left\{e_{n}: n \in \mathbb{N}\right\} \cup\{\theta\}$ where $\theta$ is the origin of $\ell_{2}$ is an $\varepsilon$-satellite system for $C$ whenever $1<\varepsilon \leq \sqrt{2}$ that has just one satellite.

LEMMA 3.3. Let $C$ be a nonempty closed subset of a metric space $\langle X, d\rangle$. Then $\nu(C) \leq \inf \{\varepsilon>0: C$ has an $\varepsilon$-satellite system $\}$.

Proof. If no $\varepsilon$-satellite system for $C$ exists for any $\varepsilon$, there is nothing to prove as the infimum of the empty set is $+\infty$. Otherwise, we show that if there is some $\varepsilon$-satellite system for $C$, then $\nu(C) \leq \varepsilon$.

By our previous remarks, we may assume there is a countably infinite $\varepsilon$-discrete subset $\left\{x_{1}, x_{2}, \ldots\right\}$ of $C$ and for each $n, y_{n} \in X$ such that for some $\delta>0$ independent of $n, \delta<d\left(x_{n}, y_{n}\right)<\varepsilon$. Fix $\lambda \in(0, \delta)$; by Zorn's lemma there is a maximal $\lambda$-discrete subset $E_{\lambda}$ of $C$ containing $\left\{x_{1}, x_{2}, \ldots\right\}$ and such that for all $x \in C, d\left(x, E_{\lambda}\right)<\lambda$. Now for each $n \in \mathbb{N}$ define $A_{n}(\lambda) \in \mathcal{C}_{0}(X)$ as follows:

$$
A_{n}(\lambda):=\left\{y_{n}\right\} \cup\left(E_{\lambda} \backslash\left\{x_{n}\right\}\right) .
$$

Since $H_{d}\left(C, E_{\lambda}\right) \leq \lambda$ and $d\left(x_{n}, y_{n}\right)<\varepsilon$, we get $H_{d}\left(C, A_{n}(\lambda)\right)<\lambda+\varepsilon$ for all $n \in \mathbb{N}$. Since $x_{n} \in A_{j}(\lambda)$ whenever $n \neq j$, we compute

$$
\begin{aligned}
H_{d}\left(A_{j}(\lambda), A_{n}(\lambda)\right) & \geq d\left(x_{n}, A_{n}(\lambda)\right)=\min \left\{d\left(x_{n}, y_{n}\right), d\left(x_{n}, E_{\lambda} \backslash\left\{x_{n}\right\}\right)\right\} \\
& \geq \min \{\delta, \lambda\}=\lambda .
\end{aligned}
$$

Thus, $\left\langle A_{n}(\lambda)\right\rangle$ can have no $H_{d}$ Cauchy subsequence and so $\left\{A: H_{d}(A, C)<\right.$ $\lambda+\varepsilon\}$ fails to have compact closure. This proves that $\nu(C) \leq \lambda+\varepsilon$, and so $\nu(C) \leq \varepsilon$ as required.

Corollary 3.4. Let $D \cup\left\{y_{x}: x \in D\right\}$ be an $\varepsilon$-satellite system for an infinite uniformly discrete set $D$ in a metric space $\langle X, d\rangle$. Then $\nu(D) \leq \varepsilon$. 
Proof. Let $D=C$ in Lemma 3.3 .

Lemma 3.5. Let $C$ be a nonempty closed subset of a metric space $\langle X, d\rangle$. Then $\nu(C) \leq \inf \{\nu(x): x \in C\}$.

Proof. If $\nu(x)=\infty$ for all $x \in C$, there is nothing to show. Otherwise let $\lambda>\inf \{\nu(x): x \in C\}$ be arbitrary and take $x_{0} \in C$ such that cl $\left(S_{d}\left(x_{0}, \lambda\right)\right)$ is not compact. Let $\alpha>\lambda$ be arbitrary. It suffices to show $\nu(C) \leq \alpha$.

Let $D$ be a maximal $\alpha$-uniformly discrete subset of $C$ containing $x_{0}$. Now either $S_{d}\left(x_{0}, \lambda\right)$ contains a Cauchy sequence $\left\langle w_{n}\right\rangle$ without a cluster point in $X$, or $S_{d}\left(x_{0}, \lambda\right)$ is not totally bounded. In the first case $\left\langle D \cup\left\{w_{n}\right\}\right\rangle$ is a sequence in the hyperspace without a cluster point where $H_{d}\left(D \cup\left\{w_{n}\right\}, C\right)$ $\leq \alpha$ for each $n$. In the second, there exists a positive $\mu<\min \{\lambda, \alpha-\lambda\}$ and $\left\{x_{1}, x_{2}, \ldots\right\} \subseteq S_{d}\left(x_{0}, \lambda\right) \backslash S_{d}\left(x_{0}, \mu\right)$ such that $n \neq j \Rightarrow d\left(x_{n}, x_{j}\right) \geq \mu$. Next for all $n \in \mathbb{N}$, let $A_{n}=D \cup\left\{x_{n}\right\}$. While $H_{d}\left(A_{n}, C\right) \leq \alpha$ for all $n$, we have $n \neq j \Rightarrow H_{d}\left(A_{n}, A_{j}\right) \geq \mu$ because $x_{0} \in D$ and $\mu<\alpha-\lambda$. This shows that $\nu(C) \leq \alpha$ as required.

We now introduce a new set-functional $\gamma: \mathcal{C}_{0}(X) \rightarrow[0, \infty]$ defined by $\gamma(C):=\sup \left\{\alpha>0: \forall \delta \in(0, \alpha), C^{\alpha} \backslash C^{\delta}\right.$ is totally bounded $\}$,

where additionally we put $\gamma(C):=0$ if no such positive $\alpha$ exists. Notice that $\gamma(C)=\infty$ whenever $X \backslash C$ is totally bounded; in particular, this is true when $C=X$ itself.

Lemma 3.6. Let $C$ be a nonempty closed subset of a metric space $\langle X, d\rangle$. Then $\nu(C) \leq \gamma(C)$.

Proof. Take $\alpha>\gamma(C)$. We intend to produce a sequence of closed sets $\left\langle A_{n}\right\rangle$ with no $H_{d^{-}}$Cauchy subsequence but such that for all $n \in \mathbb{N}$, $H_{d}\left(A_{n}, C\right)<\alpha$. To this end choose $\delta>0$ such that $C^{\alpha} \backslash C^{\delta}$ fails to be totally bounded. As a result we can find $\left\{a_{n}: n \in \mathbb{N}\right\}$ in the annulus and $\varepsilon>0$ such that whenever $n \neq j$, we have $d\left(a_{n}, a_{j}\right)>\varepsilon$. Then if we put $A_{n}=C \cup\left\{a_{n}\right\}$, we have

(i) $\forall n \in \mathbb{N}, H_{d}\left(A_{n}, C\right)<\alpha$;

(ii) whenever $n \neq j, H_{d}\left(A_{n}, A_{j}\right) \geq \min \{\varepsilon, \delta\}$.

This shows that $\left\{A \in \mathcal{C}_{0}(X): H_{d}(C, A)<\alpha\right\}$ does not have compact closure, and so $\nu(C) \leq \alpha$, as required.

Our first theorem of this section may be viewed as a quantification of Theorem 14 of [10].

TheOREM 3.7. Let $C$ be a nonempty closed subset of a metric space $\langle X, d\rangle$. Then $\nu(C)=\min \{\inf \{\varepsilon>0: C$ has an $\varepsilon$-satellite system $\}, \gamma(C)$, $\inf \{\nu(x): x \in C\}\}$. 
Proof. That $\nu(C)$ is no larger than the minimum is established by the conjunction of Lemma 3.3, Lemma 3.5, and Lemma 3.6. If the minimum is zero, there is nothing further to show. Otherwise, let $\alpha$ and $\mu$ be arbitrary positive scalars with

$$
\begin{aligned}
& \alpha+2 \mu<\min \{\inf \{\varepsilon>0: C \text { has an } \varepsilon \text {-satellite system }\}, \\
& \qquad(C), \inf \{\nu(x): x \in C\}\} .
\end{aligned}
$$

We intend to show that $\left\{A \in \mathfrak{C}_{0}(X): H_{d}(A, C) \leq \alpha\right\}$ is both complete and totally bounded with respect to Hausdorff distance.

Completeness is rather obvious, for if $H_{d}(A, C) \leq \alpha$, then $A$ is a subset of the closed set $E:=\{x: d(x, C) \leq \alpha\}$. For each $x \in E$, pick $c_{x} \in C$ with $d\left(x, c_{x}\right)<\alpha+\mu$. Since $\operatorname{cl}\left(S_{d}(x, \mu)\right) \subseteq \operatorname{cl}\left(S_{d}\left(c_{x}, \alpha+2 \mu\right)\right)$ and $\operatorname{cl}\left(S_{d}\left(c_{x}, \alpha+2 \mu\right)\right)$ is compact, $E$ viewed as a metric subspace of $X$ is uniformly locally compact and is thus a complete metric space. Thus,

$$
\left\{A \in \mathfrak{C}_{0}(X): H_{d}(A, C) \leq \alpha\right\} \subseteq \mathfrak{C}_{0}(E)
$$

and as such is effectively a closed subset of the complete metric space $\left\langle\mathrm{C}_{0}(E), H_{d}\right\rangle$.

For total boundedness, it suffices to show that the superset $\left\{A \in \mathfrak{C}_{0}(X)\right.$ : $\left.H_{d}(A, C)<\alpha+\mu\right\}$ is $H_{d}$-totally bounded. For this, we show that for each $\delta \in(0, \alpha)$, there exists a finite family $\mathcal{D}$ of closed sets such that whenever $H_{d}(A, C)<\alpha+\mu$, there exists $D \in \mathcal{D}$ with $H_{d}(A, D) \leq \delta$.

As $\alpha+\mu<\gamma(C)$ implies that $C^{\alpha+\mu} \backslash C^{\delta / 2}$ is totally bounded, we can choose a finite subset $B_{0}$ of the annulus with $C^{\alpha+\mu} \backslash C^{\delta / 2} \subseteq B_{0}^{\delta}$. By Zorn's lemma $C$ has a maximal $(\alpha+\mu)$-discrete subset $L$ such that $C \subseteq L^{\alpha+\mu}$. Since $C$ has no $(\alpha+\mu)$-satellite system,

$$
\widetilde{L}:=\left\{x \in L: S_{d}(x, \alpha+\mu) \backslash S_{d}(x, \delta / 2) \neq \emptyset\right\}
$$

is finite. Since for each $c \in C, \alpha+\mu<\nu(c)$, whenever $x \in \widetilde{L}, S_{d}(x, \alpha+\mu)$ contains a finite $\delta / 2$-net $F_{x}$. We intend to show that the finite family $\mathcal{D}$ of closed subsets of $C^{\alpha+\mu}$ defined by

$$
\mathcal{D}:=\left\{L \backslash \widetilde{L} \cup F \cup B: F \subseteq \bigcup_{x \in \widetilde{L}} F_{x} \text { and } B \subseteq B_{0}\right\}
$$

is an adequate approximating family.

Fix $A$ with $H_{d}(A, C)<\alpha+\mu$. There are two possibilities for a point $a \in A$ : either $a \in C^{\delta / 2}$, or $a \in C^{\alpha+\mu} \backslash C^{\delta / 2}$. In the first case, pick $c_{a} \in C$ with $d\left(a, c_{a}\right)<\delta / 2$. If $c_{a} \in \bigcup_{x \in \widetilde{L}} S_{d}(x, \alpha+\mu)$, pick $x \in \widetilde{L}$ and $w_{a} \in F_{x}$ with $d\left(w_{a}, c_{a}\right)<\delta / 2$, from which $d\left(w_{a}, a\right)<\delta$. Otherwise, pick $x \in L \backslash \widetilde{L}$ with $c_{a} \in S_{d}(x, \alpha+\mu)$; by the definition of $\widetilde{L}, d\left(c_{a}, x\right)<\delta / 2$ so that $d(a, x)<\delta$. In the case that $a$ lies in the annulus $C^{\alpha+\mu} \backslash C^{\delta / 2}$, pick $b_{a} \in B_{0}$ with $d\left(a, b_{a}\right)<\delta$. 
Since $H_{d}(C, A)<\alpha+\mu$ implies $L \backslash \widetilde{L} \subseteq A^{\delta / 2}$, it now follows that $D_{A} \in \mathcal{D}$ defined by $D_{A}:=L \backslash \widetilde{L} \cup\left\{w_{a}: a \in A \cap C^{\delta / 2}\right.$ and $\left.c_{a} \in \bigcup_{x \in \widetilde{L}} S_{d}(x, \alpha+\mu)\right\} \cup\left\{b_{a}\right.$ : $\left.a \in A \cap\left(C^{\alpha+\mu} \backslash C^{\delta / 2}\right)\right\}$ satisfies $H_{d}\left(A, D_{A}\right) \leq \delta$.

We note that if $C$ has totally bounded complement, then $\nu(C)$ is the minimum of the first and third quantities, whereas if $C$ itself is totally bounded, then $\nu(C)$ is the minimum of the second and third quantities.

In the context of uniform spaces, Burdick showed that if $X$ has a compact neighborhood in the hyperspace, then the hyperspace is uniformly locally compact [8] (see also Corollary 15 of [10]). This fact falls out of our formula for $\nu(C)$ as we next show.

Corollary 3.8. Let $\langle X, d\rangle$ be a metric space such that $X$ is a point of local compactness of $\left\langle\mathrm{e}_{0}(X), H_{d}\right\rangle$. Then the hyperspace is uniformly locally compact.

Proof. As we have observed, $\gamma(X)=\infty$, and so with $\zeta(C):=\min \{\inf \{\varepsilon>0: C$ has an $\varepsilon$-satellite system $\}, \inf \{\nu(x): x \in C\}\}$, we have $\nu(X)=\zeta(X)>0$. Choose $\mu>0$ such that $3 \mu<\nu(X)$. We intend to show that for all $C \in \mathrm{C}_{0}(X)$, we have $\nu(C) \geq \mu$. Since $\zeta(C) \geq \zeta(X)=\nu(X)$ and $\nu(C)=\min \{\gamma(C), \zeta(C)\}$, it suffices to show that $\gamma(C) \geq \mu$. We show that each annulus of the form $C^{\mu} \backslash C^{\delta}$, where $\delta \in(0, \mu)$, is totally bounded.

To this end, let $x_{1}$ be an arbitrary point of the annulus and choose $c_{1} \in C$ with $\delta<d\left(x_{1}, c_{1}\right)<\mu$. Now either $C^{\mu} \backslash C^{\delta} \subseteq S_{d}\left(c_{1}, 3 \mu\right)$ or not. If not, take $x_{2}$ in the annulus with $d\left(x_{2}, c_{1}\right) \geq 3 \mu$, i.e., $x_{2} \notin\left\{c_{1}\right\}^{3 \mu}$. There exists $c_{2} \in C$ with $\delta<d\left(x_{2}, c_{2}\right)<\mu$ such that $d\left(c_{2}, c_{1}\right) \geq 2 \mu$. Suppose we have chosen $x_{1}, \ldots, x_{n}$ in the annulus and $c_{1}, \ldots, c_{n}$ in $C$ with the following properties

(i) $\forall j \leq n, \delta<d\left(x_{j}, c_{j}\right)<\mu$;

(ii) whenever $i \neq j, d\left(c_{i}, c_{j}\right) \geq 2 \mu$;

(iii) $\left\{c_{1}, \ldots, c_{n}\right\}^{3 \mu}$ fails to contain $C^{\mu} \backslash C^{\delta}$.

Then we can pick $x_{n+1}$ in the annulus outside $\left\{c_{1}, \ldots, c_{n}\right\}^{3 \mu}$ and then $c_{n+1} \in C$ with $\delta<d\left(x_{n+1}, c_{n+1}\right)<\mu$, and it easily follows that for all $j \leq n, d\left(c_{j}, c_{n+1}\right) \geq 2 \mu$. Thus unless at some index we obtain $C^{\mu} \backslash C^{\delta} \subseteq$ $\left\{c_{1}, \ldots, c_{n}\right\}^{3 \mu}$, the principle of mathematical induction yields a $2 \mu$-satellite system for $C$ and hence for $X$, which contradicts the choice of $\mu$. We conclude that for some $n, C^{\mu} \backslash C^{\delta}$ is contained in a union of $n$ balls of radius of $3 \mu$, and since each ball is totally bounded, so is the annulus. This shows that $\gamma(C) \geq \mu$, completing the proof.

TheOREM 3.9. For a metric space $\langle X, d\rangle$, the following conditions are equivalent:

(1) $X$ is a point of local compactness of $\left\langle\mathfrak{C}_{0}(X), H_{d}\right\rangle$; 
(2) the hyperspace $\left\langle\mathfrak{C}_{0}(X), H_{d}\right\rangle$ is uniformly locally compact;

(3) the hyperspace $\left\langle\mathrm{e}_{0}(X), H_{d}\right\rangle$ is cofinally complete.

Proof. $(1) \Rightarrow(2)$ is Corollary 3.8 , and $(2) \Rightarrow(3)$ is well-known and easy to show. Thus only $(3) \Rightarrow(1)$ requires proof.

Assume that the hyperspace is cofinally complete. Since a closed subspace of a cofinally complete space is also cofinally complete and $x \mapsto\{x\}$ is an isometric embedding of $\langle X, d\rangle$ into $\left\langle\mathfrak{C}_{0}(X), H_{d}\right\rangle$, the underlying space is cofinally complete. If it is compact, then the hyperspace is also and there is nothing to show. Otherwise, we next claim that $\operatorname{nlc}(X)=\emptyset$.

If this is not the case, since $X$ is complete but noncompact, we conclude that $X$ must fail to be totally bounded. From this it easily follows from the compactness of nlc $(X)$ that for some $\varepsilon_{0}>0,\left\{x: d(x, \operatorname{nlc}(X))>\varepsilon_{0}\right\}$ fails to be totally bounded. Fix $p \in \operatorname{nlc}(X)$ and for some positive $\lambda$ let $\left\{x_{1}, x_{2}, \ldots\right\}$ be an infinite uniformly $\lambda$-discrete subset of $\left\{x: d(x, \operatorname{nlc}(X))>\varepsilon_{0}\right\}$. Then with $\delta=\min \left\{\varepsilon_{0}, \lambda\right\}$, we see that $\left\{p, x_{1}, x_{2}, \ldots\right\}$ is $\delta$-uniformly discrete. For each $n \in \mathbb{N}$, put $A_{n}=\left\{p, x_{n}\right\}$. By Lemma 3.5, we have $\nu\left(A_{n}\right)=0$ and clearly $\left\{A_{n}: n \in \mathbb{N}\right\}$ is a uniformly $H_{d}$-discrete set in the hyperspace. This means that $\operatorname{nlc}\left(\mathfrak{C}_{0}(X)\right)$ fails to be compact, contradicting cofinal completeness of the hyperspace.

It remains to show that if $X$ is not totally bounded and $\langle X, d\rangle$ is uniformly locally compact and the hyperspace is cofinally complete, then $\nu(X)$ is positive. If not, then since

$$
\inf \{\nu(x): x \in X\}\}>0,
$$

by Theorem 3.7 we must have

$$
\inf \{\varepsilon>0: X \text { has an } \varepsilon \text { satellite system }\}=0 .
$$

Next choose $\lambda>0$ such that for each $x \in X, \operatorname{cl}\left(S_{d}(x, \lambda)\right)$ is compact and then let $\left\{x_{1}, x_{2}, \ldots\right\}$ be an infinite $\lambda_{0}$-uniformly discrete subset of $X$ for some $\lambda_{0}<\lambda$. Pick $k \in \mathbb{N}$ with $1 / k<\lambda_{0}$, and choose, for each $n \geq k$, an $\varepsilon_{n}$-satellite system $D_{n} \cup\left\{y_{x}(n): x \in D_{n}\right\}$ where $\varepsilon_{n} \leq 1 / n$. By Corollary 3.4, we have $\nu\left(D_{n}\right) \leq 1 / n$ for all $n \geq k$.

Whenever $k \leq j<n$ define $F(j, n) \subseteq D_{n}$ as follows:

$$
F(j, n):=D_{n} \cap S_{d}\left(x_{j}, \frac{1}{2} \lambda_{0}\right) .
$$

Since $S_{d}\left(x_{j}, \frac{1}{2} \lambda_{0}\right)$ is totally bounded and $D_{n}$ is uniformly discrete, each $F(j, n)$ is finite, and again by Corollary 3.4 ,

$$
\nu\left(D_{n} \backslash \bigcup_{k \leq j<n} F(j, n)\right) \leq \frac{1}{n}
$$


because $D_{n} \backslash \bigcup_{k \leq j<n} F(j, n)$, being an infinite subset of $D_{n}$, has an $\varepsilon_{n^{-}}$ satellite system, too.

Next, set $A_{k}=D_{k} \cup\left\{x_{k}\right\}$ and for each $n>k$, put

$$
A_{n}:=\left(D_{n} \backslash \bigcup_{k \leq j<n} F(j, n)\right) \cup\left\{x_{n}\right\} .
$$

Clearly, we have $\nu\left(A_{n}\right) \leq 1 / n$ for all $n \geq k$. Further whenever $k \leq j<n$, by the form of each $F(j, n)$, we have $d\left(x_{j}, A_{n}\right) \geq \frac{1}{2} \lambda_{0}$ and so $H_{d}\left(A_{j}, A_{n}\right) \geq \frac{1}{2} \lambda_{0}$. Thus the sequence $A_{k}, A_{k+1}, A_{k+2}, \ldots$ has no $H_{d^{-}}$cluster point, which by [6, Thm. 3.4] contradicts cofinal completeness of the hyperspace. We conclude that $\nu(X)>0$, finishing the proof.

Acknowledgements. The authors are indebted to Bruce Burdick for his many helpful comments on cofinal completeness, uniform paracompactness, and hyperspaces.

\section{References}

[1] M. Atsuji, Uniform continuity of continuous functions of metric spaces, Pacific J. Math. 8 (1958), 11-16; Erratum, 941.

[2] G. Beer, Metric spaces on which continuous functions are uniformly continuous and Hausdorff distance, Proc. Amer. Math. Soc. 95 (1985), 653-658.

[3] -, More about metric spaces on which continuous functions are uniformly continuous, Bull. Austral. Math. Soc. 33 (1986), 397-406.

[4] —, UC spaces revisited, Amer. Math. Monthly 95 (1988), 737-739.

[5] —, Topologies on Closed and Closed Convex Sets, Kluwer, Dordrecht, 1993.

[6] -, Between compactness and completeness, Topology Appl. 155 (2008), 503-514.

[7] B. Burdick, On linear cofinal completeness, Topology Proc. 25 (2000), 435-455.

[8] -, Local compactness of hyperspaces, Ann. New York Acad. Sci. 704 (1993), 28-33.

[9] H. Corson, The determination of paracompactness by uniformities, Amer. J. Math. 80 (1959), 185-190.

[10] C. Costantini, S. Levi, and J. Pelant, Compactness and local compactness in hyperspaces, Topology Appl. 123 (2002), 573-608.

[11] J. Fried and Z. Frolík, A characterization of uniform paracompactness, Proc. Amer. Math. Soc. 89 (1983), 537-540.

[12] A. García-Máynez and S. Romaguera, Perfect pre-images of cofinally complete metric spaces, Comment. Math. Univ. Carolin. 40 (1999), 335-342.

[13] A. Hohti, On uniform paracompactness, Ann. Acad. Sci. Fenn. Ser. A Math. Diss. 36 (1981), 1-46.

[14] N. Howes, On completeness, Pacific J. Math. 38 (1971), 431-440.

[15] —, Modern Analysis and Topology, Springer, New York, 1995.

[16] T. Jain and S. Kundu, Atsuji spaces: equivalent conditions, Topology Proc. 30 (2006), 301-325.

[17] E. Klein and A. Thompson, Theory of Correspondences, Wiley, New York, 1984.

[18] S. Nadler and T. West, A note on Lebesgue spaces, Topology Proc. 6 (1981), 363-369.

[19] J. Rainwater, Spaces whose finest uniformity is metric, Pacific J. Math. 9 (1959), $567-570$. 
[20] M. Rice, A note on uniform paracompactness, Proc. Amer. Math. Soc. 62 (1977), 359-362.

[21] S. Romageura, On cofinally complete metric spaces, Q \& A Gen. Topology 16 (1998), 165-170.

[22] J. Smith, Review of "A note on uniform paracompactness" by Michael D. Rice, Math. Rev. 55 (1978) \# 9036.

[23] G. Toader, On a problem of Nagata, Mathematica (Cluj) 20 (1978), 77-79.

[24] W. Waterhouse, On UC spaces, Amer. Math. Monthly 72 (1965), 634-635.

Gerald Beer

Department of Mathematics

California State University Los Angeles

5151 State University Drive

Los Angeles, CA 90032, U.S.A.

E-mail: gbeer@cslanet.calstatela.edu
Giuseppe Di Maio

Dipartimento di Matematica

Facoltà di Scienze

Seconda Università degli Studi di Napoli

via Vivaldi 43

81100 Caserta, Italy

E-mail: giuseppe.dimaio@unina2.it

Received 28 September 2008;

in revised form 3 November 2009 